\title{
High Current CW Beam Profile Monitors Using Transition Radiation at CEBAF
}

\author{
P. Piot, J-C. Denard, P. Adderley, K. Capek, E. Feldl * \\ Continuous Electron Beam Accelerator Facility, 12000 Jefferson Avenue, \\ Newport News, VA 23606 \\ JUL 251930

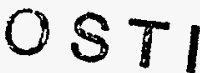

PECENGED

\begin{abstract}
One way of measuring the profile of CEBAF's low emittance and high power beam is to use the Optical Transition Radiation (OTR) emitted from a thin foil surface when the electron beam passes through it. We present the design of a monitor using the forward OTR emitted from a $0.25 \mu \mathrm{m}$ carbon foil. We believe that the monitor will resolve three main issues : $i$ ) whether the maximum temperature of the foil stays below the melting point ii) whether the beam loss remains below $0.5 \%$, in order not to trigger the machine protection system iii) and whether the monitor resolution (unlike that of synchrotron radiation monitors) is better than the product $\lambda \gamma$. It seems that the most serious limitation for CEBAF is the beam loss due to beam scattering. We present results from Keil's theory and simulations from the computer code GEANT as well as measurements with aluminum foils with a $45 \mathrm{MeV}$ electron beam. We also present a measurement of a $3.2 \mathrm{GeV}$ beam profile that is much smaller than $\lambda \gamma$, supporting Rule \& Fiorito's calculations of the OTR resolution limit due to diffraction.
\end{abstract}

\section{INTRODUCTION .}

The Optical Transition Radiation (OTR) phenomenon is a very convenient and reliable means for beam profile and position measurement in a quasi-non destructive way. However, there are limitations in the use of such monitors. Firstly, depending on the beam current of the considered beam, the screen can melt. Secondly, at high temperature, the screen becomes a source of Black Body radiation whose power can be greater than that of the OTR. Finally, the plural scattering that electrons experience while passing through the foil is a source of emittance degradation and beam loss. For the CEBAF electron beam, this last issue is of importance because of the Machine Protection System (MPS) that shuts the injector down when beam losses exceed $1 \mu A$ out of a total beam current that can reach $200 \mu A$. In this paper we describe a monitor which uses the forward OTR produced at the surface of a very thin $(0.25 \mu \mathrm{m})$ carbon foil. This device has the potential to monitor and study the continous electron beam delivered to the experimental halls.

*Work Supported by U.S. Department Of Energy contract DE-AC05-84ER40150. 


\section{DISCLAIMER}

Portions of this document may be illegible in electronic image products. Images are produced from the best available original document. 

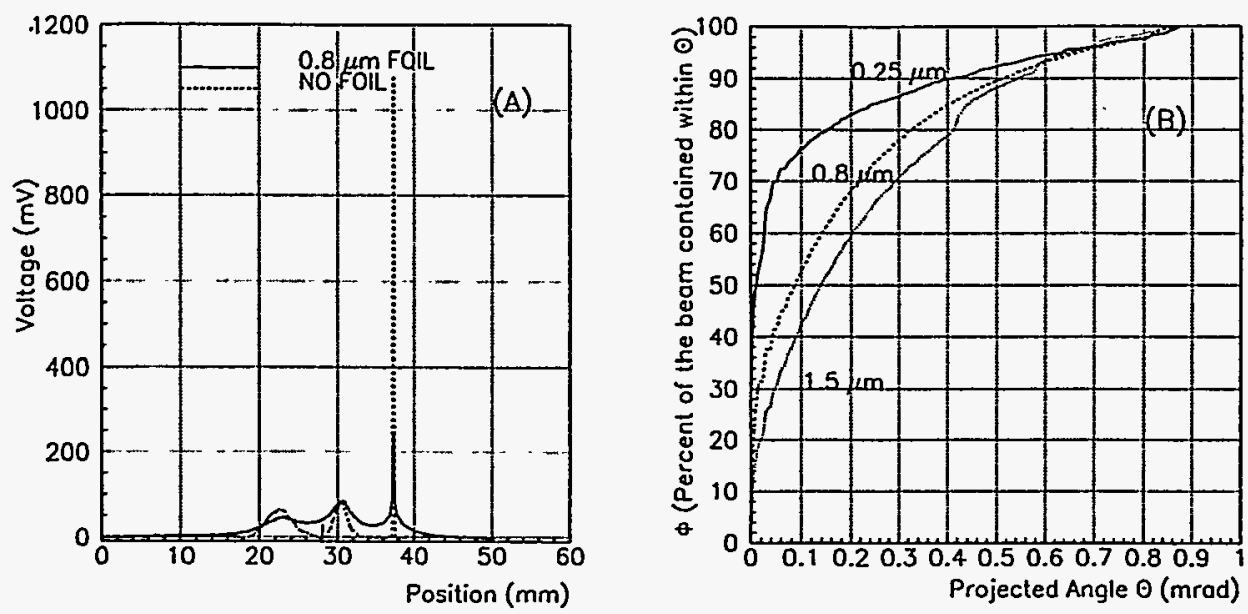

Figure. 1. Beam scattering angle distribution through thin aluminum foils, measured at $45 \mathrm{MeV}$; before inserting the foil, we set the optics for a minimum vertical beam size at the wire scanner location.(A) Wire scanner vertical profile taken $7.4 m$ after the foil set up, with and without foil; the wire scanner has three $50 \mu \mathrm{m}$ diameter wires (one vertical, one horizontal, and one at $45^{\circ}$ ).(B) Fraction of the beam contained within the vertical beam scattering angle. In order to deduce these curves from the profiles: (i) we assumed that $100 \%$ of the beam has been scanned; (ii) we considered only the right side of the third peak corresponding to the horizontal profile in order to reduce the error due to the signal received.by the other wires; (iii) we deconvoluted the relevant profiles with respect to the beam.

\section{PLURAL SCATTERING THROUGH FOILS}

\section{Experimental Results using Aluminum foils}

In this section, we present the results of the measurements of the scattering angle distribution of an electron beam through thin aluminum foils of various thicknesses, $(0.25,0.8$, and $1.5 \mu \mathrm{m})$. The foils are mounted on a support that is inserted into the beam by mean of a stepping motor. We perform this test in the $45 \mathrm{MeV}$ region of the CEBAF injector. We measure the beam size using a wire scanner installed $7.4 m$ downstream from the foils. There is no powered quadrupole magnet between the foils and the wire scanner. Therefore by knowing the distance inbetween the two instruments, we can easily calculate the distribution angle of the particles at the exit of the foil from the beam profile distribution measured by the wire scanner. The results $(F i g .1 B)$ show that, within the range of the thicknesses tested, the scattering angle containing $70 \%$ of the particles decreases in proportion with the foil thickness.

However, the angle that contains $90 \%$ of the particles does not decrease as much. Measuring angles that contain more than $90 \%$ of the beam would require 

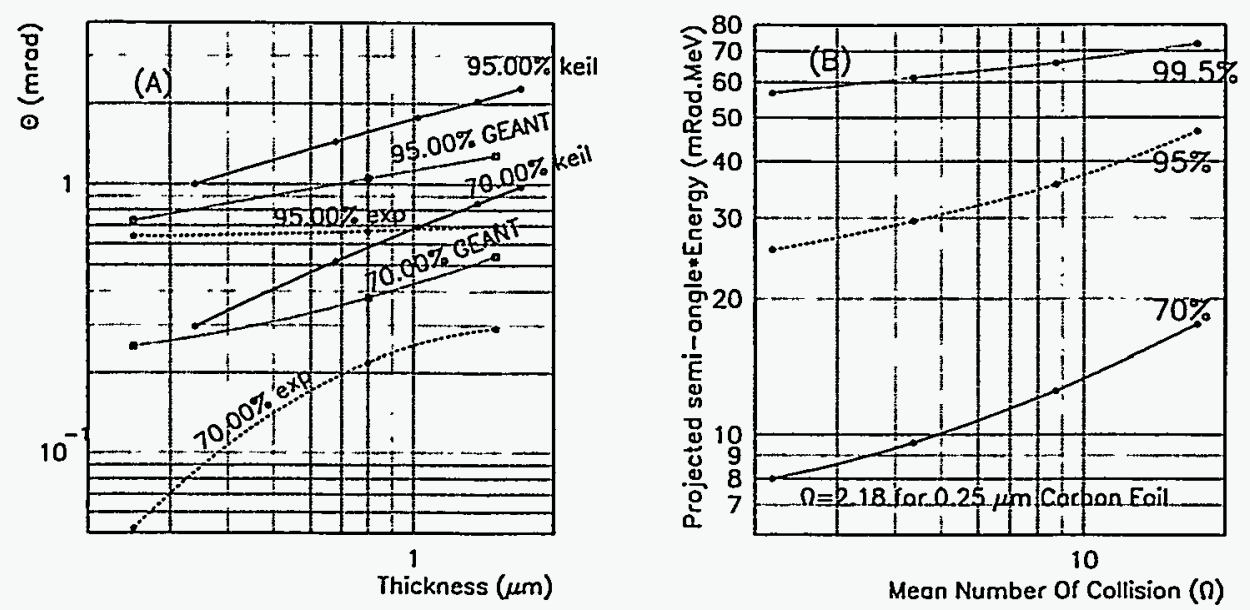

Figure. 2. (A) Comparative results of experiment with Keil's model and GEANT computation for thin aluminum foils. The curves show the effects of the foil thickness on the semi-angle containing $70 \%$ and $90 \%$ of the beam. (B) Projected Scattering angle $\Theta$ of highly relativistic electrons in thin foils according to GEANT simulation code and normalized at $1 \mathrm{GeV} . \Theta$ is the half angle at which the distribution contains the indicated percentage $\mathcal{P}$ of the beam. The projected angle of a scattered particle is defined on any plane that contains the incoming trajectory. It is the angle between the incoming and outgoing trajectory projected on that plane. The percentage of the beam loss $(1-\mathcal{P})$ can be computed at any energy for any screen material and thickness, by knowing the machine acceptance angle and the beam energy.

scanning larger angles. The curves in Fig.1 are accurate up to 90\%; beyond this value we have to use theoretical formulae or Monte-Carlo simulations.

\section{Comparative results with Keil's Model and Monte-Carlo Simulation}

In materials so thin that the mean number of collisions is less than 20 (domain of plural scattering), Molière's model of multiple scattering is not valid anymore. We used two techniques to calculate the scattering angle distribution. One is a calculation based on Keil's model(1), which is the only analytical model available. The other is a Monte-Carlo technique, GEANT(2) code. The Monte-Carlo code generated angular distribution is narrower than Keil's model by a factor of 2 . The measured distribution is narrower than the Monte-Carlo distribution by approximately another factor of 2 (Fig.2A).

The beam energy in the transport line ranges from $0.8 \mathrm{GeV}$ to $4 \mathrm{GeV}$. The beam scattering angle is inversely proportional to beam momentum. The worst case beam loss occurs at the lowest energy. At $0.8 \mathrm{GeV}$, CEBAF's beam angle 
acceptance is around $100 \mu \mathrm{rad}$ at the location we wish to install a viewer. Table 1 lists various foil materials with their smallest thickness commercially available that can be mounted on a $10 \mathrm{~mm}$ diameter hole, and the corresponding mean number of collisions $\Omega$ per $\mu m$. We used the formula:

$$
\Omega=0.78 \times \rho \times \frac{(Z+1) Z^{1 / 3}}{A}
$$

$\rho$ is the density in $\mathrm{g} / \mathrm{cm}^{3}, \mathrm{Z}$ is the atomic number, and $\mathrm{A}$ the atomic weight.

\begin{tabular}{|l|l|l|l|l|l|l|l|l|l|}
\hline Material & $\mathrm{Be}$ & $\mathrm{C}$ & $\mathrm{Mg}$ & $\mathrm{Al}$ & $\mathrm{Ti}$ & $\mathrm{Fe}$ & $\mathrm{Cu}$ & $\mathrm{Ag}$ & $\mathrm{Au}$ \\
\hline $\begin{array}{l}\text { Number Of Collis. } \\
\text { per } \mu \mathrm{m} \text { thickness }\end{array}$ & 7.5 & 8.7 & 8.3 & 13.6 & 28 & 51 & 62 & 90 & 127 \\
\hline $\begin{array}{l}\text { Thinnest foil } \\
\text { Available ( } \mu \mathrm{m}) \\
\text { for 8mm dia. } \\
\text { corresponding } \Omega\end{array}$ & 0.25 & 0.25 & 0.5 & 0.5 & 1 & 0.5 & 0.25 & 0.25 & 0.1 \\
\hline
\end{tabular}

Table 1. Survey of the materials commercially available for monitoring intense beams with OTR screens; we excluded the materials with low thermal conductivity and mean number of collisions greater than $\mathbf{3 0}$ in their smallest thickness.

The best candidates are Beryllium and Carbon; they are equivalent but we prefer the latter because of the toxicity of beryllium. From results of GEANT simulation (Fig.2B), we can estimate the percentage of beam lost at any energy for any screen material and thickness, by knowing the machine acceptance angle. Previous calculations (3) showed that aluminum foils could stand a $200 \mu \mathrm{A}$ continous beam of $50 \mu \mathrm{m}$ rms transverse dimensions. Carbon, with its equivalent thermal conductivity and higher melting point will be even superior. We present the results of a thermal analysis in the last section.

\section{OTR PROFLE MONITOR WITH A $0.25 \mu \mathrm{m}$ CARBON FOIL}

\section{Description of the Monitor}

The backward OTR depends on the reflection characteristics of the surface. There are problems with very thin foils : surface inhomogeneity makes their coefficient of reflection non uniform, and it is also difficult to stretch them enough to obtain a flat surface. Furthermore materials such as aluminum and gold present wrinkles that affect locally both reflection coefficient and angle on the scale of the beam size. These problems disappear with forward OTR since it is emitted around the beam direction regardless of the angle or coefficient of reflection of the foil. There are two additional advantages to the forward over the backward OTR with a foil at $45^{\circ}$. The depth of field error becomes negligeable. The incoming beam at normal incidence has a shorter path through 
the material which reduces the beam loss due to scattering.

We built a profile monitor, shown in Fig.3, which looks at the forward OTR emitted from a $0.25 \mu \mathrm{m}$ carbon foil mounted on a support that can be inserted into the beam. To prevent the beam from damaging the support, the support is U-shaped and open on the side crossing the beam path. A mirror sends part of the forward OTR to the optical system through a vacuum window. With the OTR that is strongly directional in a $1 / \gamma$ angular cone, we need to collect the light emitted at small angles from the beam trajectory. We did this by locating the mirror on the same insertion mechanism as the foil. The mirror is $17.5 \mathrm{~cm}$ downstream from the foil; this insertion mechanism brings its edge close to the beam trajectory $(4 \mathrm{~mm})$.

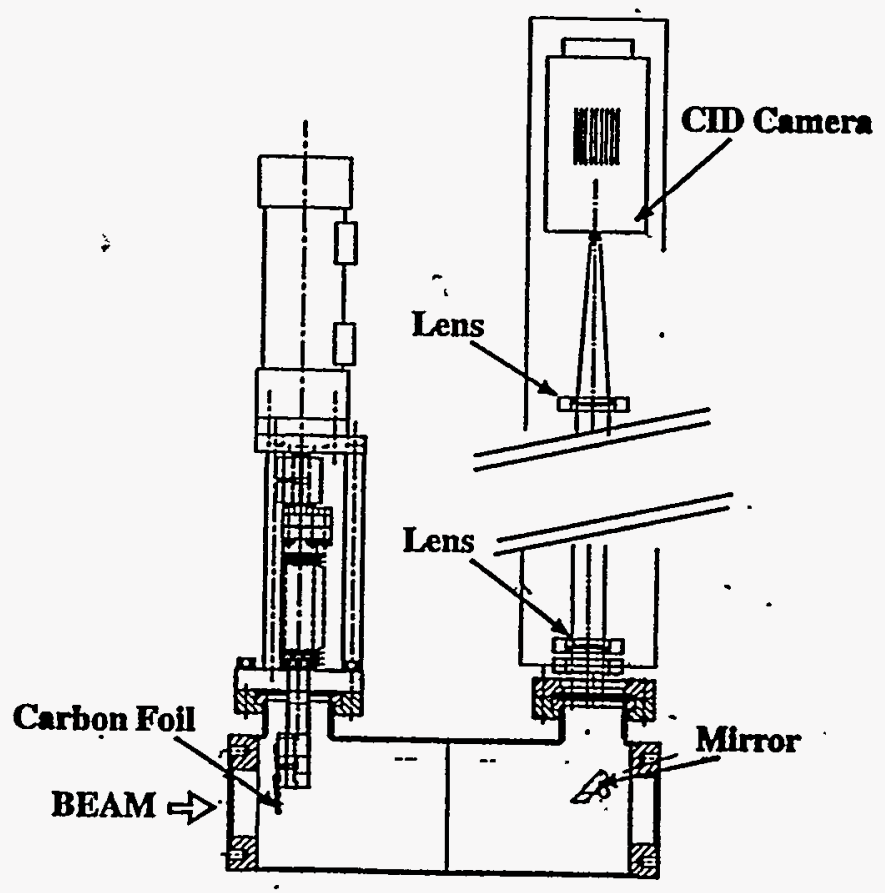

Figure. 3. Simplified view of the forward OTR Profile Monitor

The mirror collects the light and sends it to a Charge Injected Device (CID) camera via two achromatic doublets. Despite its lower sensitivity we preferred a CID camera to the more commonly used Charge Coupled Device (CCD) cameras because of its good radiation resistance (specified to $100 \mathrm{kRad}$ ). The CID array has $768 \times 575$ square pixels of $11.5 \mu m$. The camera is sensitive to the whole visible spectrum. By choosing achromatic lenses, we can improve the monitor's sensitivity without any loss in resolution. We illuminated the support from outside the vacuum chamber so that its $10 \mathrm{~mm}$ square shape can be used to precisely calibrate the CID image. The magnification of the optics is such that the $10 \mathrm{~mm}$ square support encompasses about 500 pixels which yields a pixel size of approximately $20 \mu m$ in the object plane. 


\section{Optical Resolution}

The beam profile has a gaussian shape with an rms value $\sigma \simeq 100 \mu \mathrm{m}$. We measured the resolution of the instrument in the laboratory replacing the foil with a resolution target. We found the minimum resolvable distance between two black lines to be about $80 \mu \mathrm{m}$. We believe the camera is the limiting element since this number is within a factor of 2 of the manufacturer's specifications. The resolution measured with such a target corresponds approximately to the

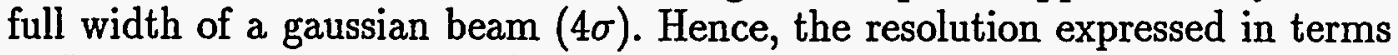
of "one sigma" is about $80 / 4=20 \mu m$, which is reasonably small compared to the expected beam size. The resolution measurement in the lab does not take into account the pupil function of the transition radiation which has a strong angular dependence. The resolution due to the OTR pupil function is a controversial issue. It has been suggested that it was limited to $1.22 \times \lambda \gamma$. However, Rule \& Fiorito calculated (4) an OTR diffraction limit very close to that of a standard source which has a uniform intensity with angle. Although the mirror center is not on the OTR revolution axis, we believe that the results reported in the following section support their calculations.

\section{Experimental Results}

We tested the monitor in the transport line of an experimental hall with a $3.2 \mathrm{GeV}$ electron beam. Figure 4 shows the horizontal beam profile measured with a $3 \mu A$ continuous beam, whose dimension is $\sigma_{x} \simeq 100 \mu m$. The camera has its maximum sensitivity at $500 \mathrm{~nm}(1.22 \lambda \gamma=3.9 \mathrm{~mm})$. The $1.22 \lambda \gamma$ product represents' the distance between the two minima of the diffraction pattern. A fit to the Airy Diffraction Function with a gaussian distribution yields a $\sigma$ that is 5.8 times smaller than the distance between the two minima of the Airy function. The hypothetical "OTR diffraction limit" at that beam energy would yield $\sigma=3.9 / 5.8=0.7 \mathrm{~mm}$ which is much greater than the $0.1 \mathrm{~mm}$ actually measured.

A test done $25 \mu \mathrm{A}$ shows that the beam scattering through the foil does not introduce losses greater than $0.5 \%$ of the beam. The electrode-sum signals of Beam Position Monitors located upstream and downstream of the foil provided the intensity information for beam loss calculation. With fluctuations of the intensity measurements at the $0.1 \%$ level, we were not able to detect any beam loss. In the near future we will test the monitor in a $180 \mu \mathrm{A}$ continuous beam to confirm that the beam loss is below the MPS threshold of $1 \mu A$.

\section{THERMAL CONDUCTION}

An electron beam going through matter deposits ionization energy that heat the foil and knocks out secondary particles. Calculation of the energy deposition (5) or its computation using the Monte-Carlo code EGS4 (6) yields similar results. The heat is lost through conduction (3) and radiation. Figure 5 shows the maximum current required to melt various materials. For $\mathrm{CE}$ - 
BAF, the maximum tolerable beam loss due to beam scattering limits actually application of OTR to lower currents.

\section{CONCLUSION}

We described an OTR profile monitor that can operate in a $200 \mu \mathrm{A}$ with continuous electron beam, using a very thin carbon foil. The measurement of a $100 \mu \mathrm{m}$ (rms radius) beam profile at $3.2 \mathrm{GeV}$ supports Rule \& Fiorito's calculations and confirms the possibility of imaging micron size beams of high energy. It also supports the concept of using the OTR monitors at CEBAF where the combination of high average beam power, small emittance, and high energy makes other profile diagnostics unsuitable. The present construction of a Free Electron Laser should be an opportunity to test this kind of monitor at higher continuous currents.

\section{REFERENCES}

1. Scott, W.T., "The theory of Small-Angle Multiple Scattering of fast Charged Particles," Rev. Mod. Phys. Vol 35 Num 2, pp. 288-290, April 1963.

2. Groosens et $\mathrm{Al}$, "GEANT, Detector Description and Simulation Tool, User Guide," CERN Application Software Group, CERN Geneva, Switzerland.

3. Denard, J-C. et Al "Experimental Diagnostics Using Optical Transition Radiation at CEBAF," in AIP Conference Proceedings 333, Beam Instrumentation Workshop, Vancouver, B.C., CANADA, October 1994.

4. Rule D.W. and Fiorito R.B., "Imaging Micron-Sized Beams with Optical Transition Radiation," in Particles and Fields Series 44, Accelerator Instmentation, 2nd Annual Workshop, Batavia, II, 1990, p315.

5. Fisher, C., Private Communication, November 1995.

6. Nelson, W.R., Hirayama, H., Rogers, D.W.O., "The EGS4 Code System," Standford Linear Accelerator Center report SLAC-210, 1978. 


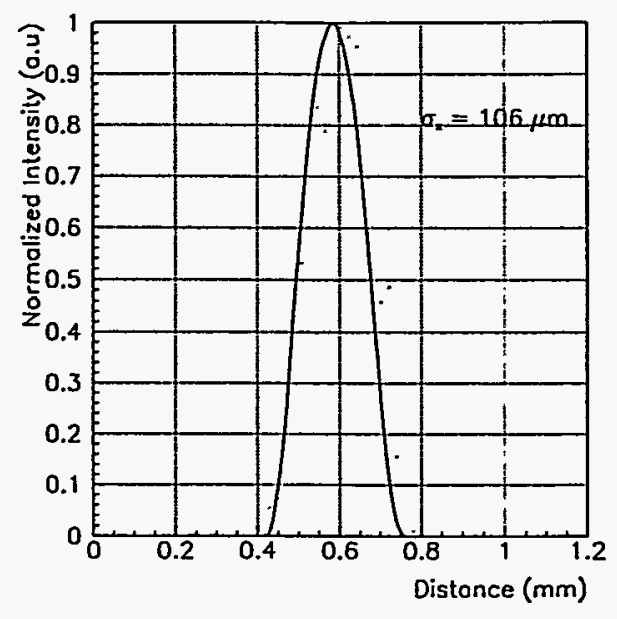

Figure. 4. Horizontal profile of a $3.2 \mathrm{GeV}, 3 \mu \mathrm{A}$ beàm measured with the OTR monitor.

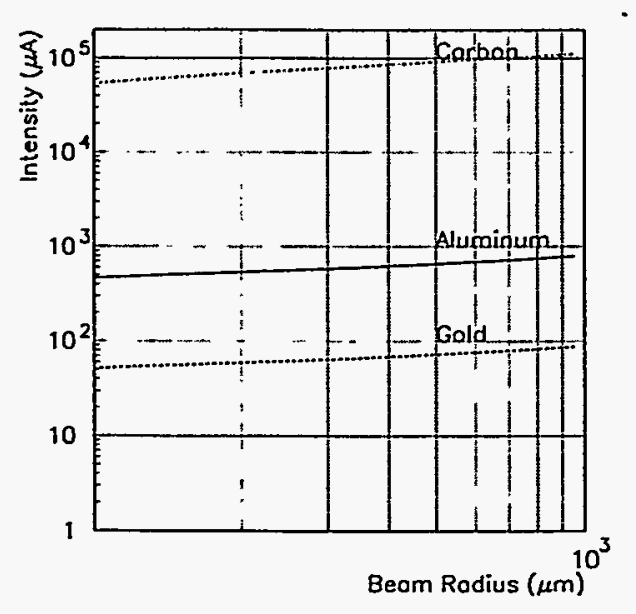

Figure. 5. Beam intensity and radius that bring various foil materials to their melting point, taking conduction and radiation phenomena into account. The calculation considers $25 \mathrm{~mm}$ diameter foils whose outside edge is at ambient temperature, a reasonable assumption for small energy deposition. 